\title{
一般化確率微分方程式の状態推定問題人の応用
}

\author{
秋月影雄・簮川徹史 \\ 早稲田大学理工学部 東京都新宿区西大久保 4-170 \\ (昭和 46 年 6 月 8 日 受付)
}

\section{One Application of Generalized Stochastic Differential Equations to the Filtering Problem}

\author{
Kageo Akizuki and Tetsushi Sasagawa \\ (School of Science and Engineering, Waseda University, Shinjuku-ku, Tokyo)
}

(Received June 8, 1971)

In recent years there has been a great deal of activity in the study of estimation theory. It is the work of Kalman and Bucy that laid the foundation of this development. In Kalman-Bucy method, it is assumed that the sample paths of the message process and the observed process are continuous in $t$. Namely, it is supposed that the signal and the observation are generated by Ito's equations, more intuitively, the dynamical system and the observation system are corrupted by white noise. But in this paper, we assume that these systems are disturbed by constantly operating noise continuous in $t$ and disturbances which cause random shocks or jumps in random time.

The object of this paper is to show that it is possible to apply the theory of generalized stochastic differential equations to studying dynamical systems corrupted by additive noise of which sample paths are not continuous in $t$ but continuous in probability.

Also, it is very interesting to study qualitative properties of solutions of generalized stochastic differential equations. In fact, some efforts have been devoted to the discussion of the stability properties of the solution process and to the study of first exit times or, equivalently, to the problem of obtaining useful upper bounds to the probability that the state of the process will leave some given set at least once by a given time.

These investigations are closely related to the problem of stochastic controllability. However, this problem is fairly difficult and scarcely any result has ever been obtained. Therefore, the discussion in this paper is restricted to study only the optimal filtering problem based on KalmanBucy's theory. Consequently, this problem is reduced to solve the nonlinear differential equation governing the covariance matrix of the errors of the best linear estimate, called the matrix Riccati equation and we try to integrate this fundamental equation approximately based on perturbation theory.

\section{1. 緒言}

状態推定問題は, Kalman-Bucy の論文 ${ }^{1)}$ が提出さ れて以来, 活発に研究されてきた. この方法では, モ デル化されたシステムが伊藤形の線形確率微分方程式
によって記述されている。すなわち，より直観的には， 力学系および観測機構は正規性白色雑音によって乱さ れているものとしている. この結果, 力学系の出力 （状態）㔚よび観測量は，すべての $t$ 亿関して連続と なる、しかしながら，実際のシステムが受ける外乱は， 必ずしも時刻 $t$ 亿関して連続ではなく, 衝撃的に加わ 
るものも多いと思われる，そこで, 本論文では, 力学 系特よび観測機構が不規則な時刻に不規則な衝撃を受 け，状態括よび観測量が飛躍的な変化を生ずる場合を 考慮したシステムの推定問題を取り扱う、本論文でる 状態推定の問題を Kalman の場合と同梯に, 平均 2 乗誤差を最小炡るるのとしてとらえているが，

Kalman の場合と大きく異なる点は, 状態と観測はと もに $t$ 汇関して連続ではなく, 確率連続となる場合を 取り扱っていることである.

\section{2. 一般化確率微分方程式}

(1·1) 式の確率微分方程式は一般化確率微分方程式 と呼ばれている.

$$
\begin{aligned}
d X(t)= & A(t) X(t) d t+R(t) d B(t) \\
& +\int_{R^{n}} C(t, u) \tilde{N}(d t, d u)
\end{aligned}
$$

ここで, 右辺の第 3 項がない場合がいわゆる伊藤形の 確率微分方程式である. 直観的にい光ば, 右辺の第 2 項がウィーナー過程に対応する項で, 第 3 項がポアッ ソン過程に対応する，大ざっぱにいって，独立增分を もつ確率連続な過程は互いに独立なウィーナー過程と ポアッソン過程の和として表わされるから(1.1) 式は 十分広い範囲の確率連続な加法過程を含んでいるもの 々思われる. 方程式 (1.1) 式自身関する数学的な詳 しい議論はたと兄ば文献 (3)，(4)，(5) に述べられてい るのでここでは省略する.以下では方程式の係数 $A(t)$, $R(t), C(t, u)$ 你解の存在と一意性を保証する程度の 条件はみたしているものと仮定する，このとき，方程 式 (1-1) 式は, 第 2 種の不連続性を持たず, 確率 1 をる って有界な解をるち, さらに $E\left[\left\|X_{0}\right\|^{2}\right]<\infty$ ならば, すべての $t \in\left[t_{0}, T\right]$ 亿対して $E\left[\|X(t)\|^{2}\right]<C$ (定数). したがって，解過程 $X(t)$ は $\left[t_{0}, T\right]$ 上でヒルベルト 空間を構成している。 また，同じ初期条件をもつこの 方程式の任意の 2 つの解は，これらの過程が連続であ るすべての $t$ の点で確率 1 をるって一致する，過程 $X(t)$ は確率連続である.

(1·1) 式に対して次の定理が成り立つ.

【定理 1】『g(t,X) が $\Pi(d u)$ と $\tilde{N}(t, d u)$ 飞関 して可積分でありかつすべての $t \in\left[t_{0}, T\right]$ と $X \in R^{n}$ 飞対して連続な導関数 $g_{t}{ }^{\prime}(t, X), g_{X}{ }^{\prime}(t, X), g_{X X^{\prime}}{ }^{\prime \prime}(t$, $X)$ をるつとする。このとき，

$$
\begin{aligned}
d g(t, X)= & g_{t^{\prime}}(t, X)+\langle\nabla g(t, X), A(t) X\rangle \\
& +\langle\nabla g(t, X), R(t) d B(t)\rangle \\
& +\frac{1}{2} s p\left(\nabla^{2}(g(t, X)) R(t) R^{*}(t)\right) \\
& +\int_{R^{n}}[g(t, X+C(t, u))-g(t, X) \\
& -\langle\nabla g(t, X), C(t, u)\rangle] \Pi(d u) \\
& +\int_{E^{n}}[g(t, X+C(t, u)) \\
& -g(t, X)] \widetilde{N}(d t, d u) 』
\end{aligned}
$$

ここで, $\nabla g(t, X)$ は成分が $g_{x k}{ }^{\prime}(t)$ のベクトル,

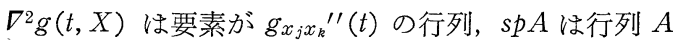
の跡, $R^{*}(t)$ は行列 $R(t)$ の転置, 〈·, ·〉はベクトル の内積を表わす。

\section{3. 状態推定問題}

まず，以下で扱う問題を正確仁訹しょう。外乱を 受けているシステムは線形であるとし，その状態べク トル $X(t)$ は次の一般化確率微分方程式,

$$
\begin{aligned}
d X(t)= & A(t) X(t) d t+R(t) d B_{1}(t) \\
& +\int_{R^{n}} C\left(t, u_{1}\right) \widetilde{N}_{1}\left(d t, d u_{1}\right)
\end{aligned}
$$

を満足するものとする。ただし， $X(t): n \times 1$ 状態べ クトル, $A(t): n \times n$ 行列, $R(t): n \times r$ 行列, $C\left(t, u_{1}\right)$ $: n \times 1$ ベクトル, $B_{1}(t):$ 各成分が独立な $r$ 次元ウィ 一ナ一過程, $\tilde{N}_{1}(t, A)=N_{1}(t, A)-t \Pi_{1}(A), N_{1}(t, A)$ : パラメータ $t \Pi_{1}(A)$ をるつポフッソン測度, 状態 $X(t)$ の観測は雑音を含んだもののみが得られるとし, 観測 ベクトル $Z(t)$ として状態の線形結合,

$$
\begin{aligned}
d Z(t)= & H(t) X(t) d t+Q(t) d B_{2}(t) \\
& +\int_{R k} D\left(t, u_{2}\right) \widetilde{N}_{2}\left(d t, d u_{1}\right)
\end{aligned}
$$

を考学る.ここで, $Z(t): k \times 1$ 観測ベクトル， $H(t)$ $: k \times n$ 行列, $Q(t): k \times m$ 行列, $D\left(t, u_{2}\right): k \times 1 \sim$ クトル, $B_{2}(t)$ : 各成分が独立な $m$ 次元ウィーナ一過 程, $\widetilde{N}_{2}(t, A)=N_{2}(t, A)-t \Pi_{2}(A), \quad N_{2}(t, A)$ : パラ メータ $t \Pi_{2}(A)$ をるつポアッソン測度.

また, $B_{1}(t), B_{2}(t), \tilde{N}_{1}(t, A), \tilde{N}_{2}(t, A)$ は互いに独 立であると仮定し，雑音の性質を次のように規定する

(いずれも平均值は零とする)、すなわち， $\Psi(s), \Lambda(s$, $u)$ をとれぞれ $d B(s), \widetilde{N}(d t, d u)$ 亿関して可積分な任 意関数とするとき，

$$
\begin{gathered}
\operatorname{cov}\left[\int_{t_{0}}^{t} \Psi(s) d B_{i}+\int_{t_{0}}^{t} \int_{R_{i}} \Lambda\left(s, u_{i}\right) \widetilde{N}_{i}\left(d s, d u_{i}\right), \int_{t_{0}}^{t} \Psi(s) d B_{i}(s)+\int_{t_{0}}^{t} \int_{R_{i}} \Lambda\left(s, u_{i}\right) \widetilde{N}_{i}\left(d s, d u_{i}\right)\right] \\
=\int_{t_{0}}^{t} \Psi(s) \Sigma_{i} \Psi^{*}(s) d s+\int_{t_{0}}^{t} \int_{R_{i}} \Lambda\left(s, u_{i}\right) V_{i} \Lambda^{*}\left(s, u_{i}\right) \Pi_{i}\left(d u_{i}\right) d s \quad(i=1,2)
\end{gathered}
$$

（ただし， $R_{1}=R^{n}, R_{2}=R^{k} ; \sum_{i}, V_{i}$ は定数行列である). 
ところで，われわれが状態推定の問題と最適制御の 問題を同時に考える場合には，(2・1) 式はとらぜんこ ントロールの項を含むべきであるが，ここで扱う問題 はシステムが線形であり, 最適制御に対する評価関数 を2次形式とすれば，よく知られている分離定理 (seperation theorem) が成り立つことが予想される. したがって, 以下では一般性を失らことなく最適制御 問題と状態推定問題を分離し，(2・1) 式はコントロー ルの項を含まないものとして㧧く．

さて, ここで取り扱ら問題を正確に定式化すると次 のよらになる。

\section{『状態推定問題,}

$t_{0} \leq s \leq t$ に対して $Z(s)$ が与えられたとき，すべて の $X^{*}$ に対し,

$$
E\left[\left\langle X^{*}, X(t)-\hat{X}(t \mid t)\right\rangle^{2}\right], X^{*} \in \mathcal{H}^{*}=\mathscr{H}
$$

を最小にするような推定值 $\hat{X}(t \mid t)$ を,

$$
\hat{X}(t \mid t)=\int_{t_{0}}^{t} L(t, s) d Z(s)
$$

なる形式で決定せよ。

ただし， $L(\cdot, \cdot)$ は両方の変数について可積分であり，

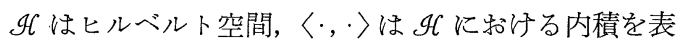
わす!. $\nearrow \hat{X}(t \mid t)$ が状態推定問題の解となるための必要十分 条件は次の定理によって与えられる2.

定理 2 『 $\hat{X}(t \mid t)$ が状態推定問題の解であるため の必要十分条件は, すべての $\sigma, \tau\left(t_{0} \leq \tau<\sigma \leq t\right)$ に対 ᄂ,

$$
\begin{aligned}
\operatorname{cov} & {[X(t), Z(\sigma)-Z(\tau)] } \\
& =\operatorname{cov}\left[\int_{t_{0}}^{t} L(t, s) d Z(s), Z(\sigma)-Z(\tau)\right]
\end{aligned}
$$

が成立することである.』

ここで, $(2 \cdot 1)$ 式の解を与えて扣こう，いま，常微 分方程式 $d / d t X(t)=A(t) X(t)$ の基本解行列を $\Phi(t$, $\left.t_{0}\right)$ とすれば, 定理 1 を用いて, $(2 \cdot 1)$ 式の解,

$$
\begin{aligned}
X(t)= & \Phi\left(t, t_{0}\right)\left[X\left(t_{0}\right)+\int_{t_{0}}^{t} \Phi\left(t_{0}, s\right) R(s) d B_{1}(s)\right. \\
& \left.+\int_{t_{0}}^{t} \int_{R^{n}} \Phi\left(t_{0}, s\right) C\left(s, u_{1}\right) \widetilde{N}_{1}\left(d s, d u_{1}\right)\right]
\end{aligned}
$$

を得る. 以下, Kalman 流の考え方により議論を進め よう.

【補題 1】「すべての $t$ に対し, $\operatorname{cov}\left[B_{1}(t), X\left(t_{0}\right)\right]$ $=0, \operatorname{cov}\left[\tilde{N}_{1}(t, A), X\left(t_{0}\right)\right]=0$ 拉よび $L(t, s)$ が $d B_{1}$ ( $t)$ と $\widetilde{N}_{1}\left(d t, d u_{1}\right)$ に関して可積分であると仮定しょ う.このとき,

$$
\begin{aligned}
\operatorname{cov} & {\left[\int_{t_{0}}^{t} L(t, s) d B_{1}(s)+\int_{t_{0}}^{t} \int_{R^{n}} L(t, s) C\left(s, u_{1}\right) \tilde{N}_{1}\left(d s, d u_{1}\right), Z(\sigma)-Z(\tau)\right] } \\
& =\int_{t_{0}}^{\sigma} L(t, s) \Sigma_{1} R^{*}(s) \phi_{\sigma}{ }^{*}(s) d s+\int_{t_{0}}^{\sigma} \int_{E^{n}} L(t, s) C\left(s, u_{1}\right) V_{1} C^{*}\left(s, u_{1}\right) \phi_{\sigma} *(s) \Pi_{1}\left(d u_{1}\right) d s
\end{aligned}
$$

ここで,

$$
\phi_{o}(s)=\int_{s}^{\sigma} H(p) \Phi(p, s) d p
$$

これは, $(2 \cdot 2)$ 式をてからのまで積分し, 解 (2.6) 式を代入した後, (2.7) 式の左辺を計算すれば直接得られ

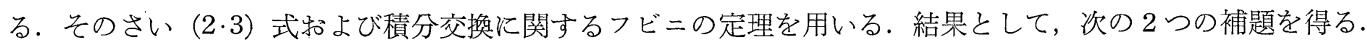

【補題 2】 $\Gamma L(t, s)$ が $t$ に関して連続的微分可能ならば， $\sigma<t$ に対して，

$$
\begin{aligned}
& \frac{d}{d t} \operatorname{cov}\left[\int_{t_{0}}^{t} L(t, s) d B_{1}(s)+\int_{t_{0}}^{t} \int_{R^{n}} L(t, s) C\left(s, u_{1}\right) \widetilde{N}_{1}\left(d s, d u_{1}\right), Z(\sigma)-Z(\tau)\right] \\
& \quad=\operatorname{cov}\left[\int_{t_{0}}^{t} \frac{\partial}{\partial t} L(t, s) d B_{1}(s)+\int_{t_{0}}^{t} \int_{R^{n}}\left(\frac{\partial}{\partial t} L(t, s)\right) C\left(s, u_{1}\right) \widetilde{N}_{1}\left(d s, d u_{1}\right), Z(\sigma)-Z(\tau)\right]
\end{aligned}
$$

【補題 3】「すべての $t$ に対して $\operatorname{cov}\left[B_{2}(t), X\left(t_{0}\right)\right]=0, \operatorname{cov}\left[N_{2}(t, A), X\left(t_{0}\right)\right]=0$ とする. このとき， $\sigma<t$ に対して,

$$
\frac{d}{d t} \operatorname{cov}[X(t), Z(\sigma)-Z(\tau)]=\operatorname{cov}[A(t) X(t), Z(\sigma)-Z(\tau)]
$$

以下では, $X\left(t_{0}\right)$ と $B_{1}(t), B_{2}(t), \widetilde{N}_{1}(t, A), \widetilde{N}_{2}(t, A)$ は常に独立であると仮定して持く. すると次の補題を得 る.

【補題 4】「L(t,s) が $d Z(s)$ に関して可積分であると仮定する。このとき，

$$
\begin{aligned}
& \operatorname{cov}\left[\int_{t_{0}}^{t} L(t, s) d Z(s), Z(\sigma)-Z(\tau)\right]=\int_{t_{0}}^{t} L(t, s) H(s) \Phi\left(s, t_{0}\right) d s \cdot \operatorname{cov}\left[X\left(t_{0}\right), X\left(t_{0}\right)\right] \cdot \int_{\tau}^{\sigma} \Phi^{*}\left(s, t_{0}\right) H^{*}(s) d s \\
& +\int_{t_{0}}^{t} \Psi(t, s) R(s) \Sigma_{1} R^{*}(s) \phi_{\sigma}^{*}(s) d s+\int_{t_{0}}^{t} \int_{R^{n}} \Psi(t, s) C\left(s, u_{1}\right) V_{1} C^{*}\left(s, u_{1}\right) \phi_{\sigma}^{*}(s) \Pi_{1}\left(d u_{1}\right) d s
\end{aligned}
$$


$+\int_{\tau}^{\sigma} L(t, s) Q(s) \sum_{2} Q^{*}(s) d s+\int_{\tau}^{\sigma} \int_{R k} L(t, s) D\left(s, u_{2}\right) V_{2} D^{*}\left(s, u_{2}\right) \Pi\left(d u_{2}\right) d s$ ただし，

$$
\Psi(t, s)=\int_{s}^{t} L(t, b) H(b) \Phi(b, s) d b
$$

【証明】次のことに注意しよう。

$$
\begin{aligned}
& \int_{t_{0}}^{t} L(t, s) d Z(s)=\int_{t_{0}}^{t} L(t, s) H(s) X(s) d s+\int_{t_{0}}^{t} L(t, s) Q(s) d B_{2}(s)+\int_{t_{0}}^{t} \int_{R k} L(t, s) D\left(s, u_{2}\right) \widetilde{N}_{2}\left(d s, d u_{2}\right) \\
& \quad=\int_{t_{0}}^{t} L(t, s) H(s)\left[\Phi\left(s, t_{0}\right) X\left(t_{0}\right)+\int_{t_{0}}^{s} \Phi(s, p) R(p) d B_{1}(p)+\int_{t_{0}}^{s} \int_{R^{n}} \Phi(s, p) C\left(p, u_{1}\right) \widetilde{N}_{1}\left(d p, d u_{1}\right)\right] d s \\
& \quad+\int_{t_{0}}^{t} L(t, s) Q(s) d B_{2}(s)+\int_{t_{0}}^{t} \int_{R k} L(t, s) D\left(s, u_{2}\right) \widetilde{N}_{2}\left(d s, d u_{2}\right)
\end{aligned}
$$

初期値 $X\left(t_{0}\right)$ および雑音が互いに独立であることとフビニの定理，さらに補題 1 を用いれば，(2.11）式が得ら れる. (証明終)

この結果として容易に得られる補題を証明なしに与えておく.

【補題 5】「L(t,s) が $t$ 亿関して連続的微分可能ならば, $\sigma<t$ に対して,

$$
\begin{aligned}
& \frac{d}{d t} \operatorname{cov}\left[\int_{t_{0}}^{t} L(t, s) d Z(s), Z(\sigma)-Z(\tau)\right]=\operatorname{cov}\left[\int_{t_{0}}^{t} \frac{\partial}{\partial t} L(t, s) d Z(s), Z(\sigma)-Z(\tau)\right] \\
& \quad+\operatorname{cov}[L(t, t) H(t) X(t), Z(\sigma)-Z(\tau)]
\end{aligned}
$$

以上の補題をもとにして次の定理を得る.

【定理 3】『tに関して連続的微分可能な $L(t, s)$ に対して,

$$
\hat{X}(t \mid t)=\int_{t_{0}}^{t} L(t, s) d Z(s)
$$

の形式の状態推定問題の解が存在すると仮定する. このとき， $t_{0} \leq s \leq t$ に対して，

$$
\frac{\partial}{\partial t} L(t, s)=A(t) L(t, s)-L(t, t) H(t) L(t, s)
$$

【証明】 $\hat{X}(t \mid t)$ は状態推定問題の解であるから，(2.5) 式によって,

$$
\frac{d}{d t} \operatorname{cov}[X(t), Z(\sigma)-Z(\tau)] \equiv \frac{d}{d t} \operatorname{cov}\left[\int_{t_{0}}^{t} L(t, s) d Z(s), Z(\sigma)-Z(\tau)\right]
$$

また, (2·10), (2·13) 式から，

したがって，

$$
\begin{aligned}
& \operatorname{cov}\left[A(t) \int_{t_{0}}^{t} L(t, s) d Z(s), Z(\sigma)-Z(\tau)\right] \equiv \operatorname{cov}\left[\int_{t_{0}}^{t} \frac{\partial}{\partial t} L(t, s) d Z(s), Z(\sigma)-Z(\tau)\right] \\
& \quad+\operatorname{cov}\left[L(t, t) H(t) \int_{t_{0}}^{t} L(t, s) d Z(s), Z(\sigma)-Z(\tau)\right]
\end{aligned}
$$

$$
\operatorname{cov}\left[\int_{t_{0}}^{t}\left[A(t) L(t, s)-\frac{\partial}{\partial t} L(t, s)-L(t, t) H(t) L(t, s)\right] d Z(s), Z(\sigma)-Z(\tau)\right]=0
$$

いま，

$$
\mathcal{L}(t, s) \equiv A(t) L(t, s)-\frac{\partial}{\partial t} L(t, s)-L(t, t) H(t) L(t, s)
$$

と扰く.(2·15) 式から，

$$
\int_{t_{0}}^{t}[L(t, s)+\mathcal{L}(t, s)] d Z(s)=\hat{Y}(t \mid t)
$$

もまた (2.5) 式を満たし，したがって最適フィルタである. 射影定理により，すべての $X^{*} に$ に対して，

$$
E\left[\left\langle X^{*}, \hat{Y}(t \mid t)-\hat{X}(t \mid t)\right\rangle^{2}\right]=0
$$

すなわち，すべての $X^{*}$ に対して，

$$
X * \operatorname{cov}\left[\int_{t_{0}}^{t} \mathcal{L}(t, s) d Z(s), \int_{t_{0}}^{t} \mathcal{L}(t, s) d Z(s)\right] X=0
$$

ところで, 


$$
\begin{aligned}
& \operatorname{cov}\left[\int_{t_{0}}^{t} \mathcal{L}(t, s) d Z(s), \int_{t_{0}}^{t} \mathcal{L}(t, s) d Z(s)\right]=\operatorname{cov}\left[\int_{t_{0}}^{t} \mathcal{L}(t, s) H(s) X(s) d s, \int_{t_{0}}^{t} \mathcal{L}(t, s) H(s) H(s) X(s) d s\right] \\
& \quad+\int_{t_{0}}^{t} \mathcal{L}(t, s) Q(s) \Sigma_{2} Q^{*}(s) \mathcal{L}^{*}(t, s) d s+\int_{t_{0}}^{t} \int_{R k} \mathcal{L}(t, s) D\left(s, u_{2}\right) V_{2} D^{*}\left(s, u_{2}\right) \mathcal{L}^{*}(t, s) \Pi_{2}\left(d u_{2}\right) d s
\end{aligned}
$$

ここで, $Q(s) \Sigma_{2} Q^{*}(s)$ 搗よび $\int_{R k} D\left(s, u_{2}\right) V_{2} D^{*}\left(s, u_{2}\right) \Pi_{2}\left(d u_{2}\right)$ がすべての $s$ について正定值行列であると仮 定すれば， $\mathcal{L}(t, s)=0$ をえる. したがって定理が証明された.

【定理 4】『推定值 $\hat{X}(t \mid t)$ は次の方程式,

$$
\begin{aligned}
& d \hat{X}(t \mid t)=[A(t)-K(t) H(t)] \hat{X}(t \mid t) d t+K(t) H(t) X(t) d t+K(t) Q(t) d B_{2}(t) \\
& \quad+K(t) \int_{R k} D\left(t, u_{2}\right) \widetilde{N}_{2}\left(d t, d u_{2}\right)
\end{aligned}
$$

をみたす．ただし， $K(t)=L(t, t)$ は $t$ に関して連続的微分可能とする.

また, $e(t)=X(t)-\hat{X}(t \mid t)$ と和けば,

$$
\begin{aligned}
d e(t)= & {[A(t)-K(t) H(t)] e(t) d t+R(t) d B_{1}(t)-K(t) Q(t) d B_{2}(t)+\int_{R^{n}} C\left(t, u_{1}\right) \tilde{N}_{1}\left(d t, d u_{1}\right) } \\
& -\int_{R^{k}} K(t) D\left(t, u_{2}\right) \tilde{N}_{2}\left(d t, d u_{2}\right)
\end{aligned}
$$

である・』

\section{【証明】}

$$
\hat{X}(t \mid t)=\int_{t_{0}}^{t} L(t, s) d Z(s)=\int_{t_{0}}^{t} L(t, s) H(s) X(s) d s+\int_{t_{0}}^{t} L(t, s) Q(s) d B_{2}(s)+\int_{t_{0}}^{t} \int_{R k} L(t, s) D\left(s, u_{2}\right) \tilde{N}_{2}\left(d t, d u_{2}\right)
$$

これを用いて, $(2 \cdot 16)$ 式の第 1 項を計算する.

$$
\begin{aligned}
& \int_{t_{0}}^{t}[A(s)-K(s) H(s)] \hat{X}(s \mid s) d s=\int_{t_{0}}^{t}[A(s)-K(s) H(s)] \cdot \int_{t_{0}}^{s} L(s, p) H(p) X(p) d p \cdot d s \\
& \quad+\int_{t_{0}}^{t}[A(s)-K(s) H(s)] \cdot \int_{t_{0}}^{s} L(s, p) Q(p) d B_{2}(p) \cdot d s \\
& \quad+\int_{t_{0}}^{t}[A(s)-K(s) H(s)] \cdot \int_{t_{0}}^{s} \int_{R k} L(s, p) D\left(p, u_{2}\right) \tilde{N}_{2}\left(d p, d u_{2}\right) d s
\end{aligned}
$$

ここで, フビニの定理を用いて積分順序を交換すれば,

$$
\begin{aligned}
= & \int_{t_{0}}^{t}\left\{\int_{p}^{t}[A(s)-K(s) H(s)] L(s, p) d s\right\} H(p) X(p) d p+\int_{t_{0}}^{t}\left\{\int_{p}^{t}[A(s)-K(s) H(s)] L(s, p) d s\right\} Q(p) d B_{2}(p) \\
& +\int_{t_{0}}^{t} \int_{R k}\left\{\int_{p}^{s}[A(s)-K(s) H(s)] L(s, p) d s\right\} D\left(p, u_{2}\right) \widetilde{N}_{2}\left(d p, d u_{2}\right)
\end{aligned}
$$

さらに $(2 \cdot 14)$ 式を用いて,

$$
\int_{t_{0}}^{t}[A(s)-K(s) H(s)] \hat{X}(s \mid s) d s=\hat{X}(t \mid t)-\int_{t_{0}}^{t} K(s) d Z(s)
$$

をえる.これで $(2 \cdot 16)$ 式が得られた. (2·16) 式から, (2·17) 式を導くことは容易である」.

この定理に和ける $K(t)$ は次の定理によって与えられる.

【定理 5】『リッカチ形の微分方程式,

$$
\begin{aligned}
\dot{P}(t)= & A(t) P(t)+P(t) A^{*}(t)-P(t) H^{*}(t)\left[Q(t) \Sigma_{2} Q^{*}(t)+\int_{R k} D\left(t, u_{2}\right) V_{2} D^{*}\left(t, u_{2}\right) \Pi_{2}\left(d u_{2}\right)\right]^{-1} H(t) P(t) \\
& +R(t) \Sigma_{1} R^{*}(t)+\int_{R^{n}} C\left(t, u_{1}\right) V_{1} C^{*}\left(t, u_{1}\right) \Pi_{1}\left(d u_{1}\right) \\
P\left(t_{0}\right)= & P_{0}=\operatorname{cov}\left[X\left(t_{0}\right), X\left(t_{0}\right)\right] .
\end{aligned}
$$

の解を $P(t)$ とするとき,

$$
K(t)=P(t) H^{*}\left[Q(t) \Sigma_{2} Q^{*}(t)+\int_{R k} D\left(t, u_{2}\right) V_{2} D^{*}\left(t, u_{2}\right) \Pi_{2}\left(d u_{2}\right)\right]^{-1}
$$

である』.

この定理の証明は容易ではあるがて長であるので, 結果のみにとどめて特こう. 


\section{4. リッカチ形非線形微分方程式の近似解}

ここでは，前章で得られた主要な方程式であるリッ カチ形の非線形方程式,

$$
\begin{aligned}
& \dot{P}(t)=A(t) P(t)+P(t) A^{*}(t) \\
& \quad-P(t) H^{*}(t) M^{-1}(t) H(t) P(t)+N(t) \\
& P\left(t_{0}\right)=P_{0}
\end{aligned}
$$

ただし，

$$
\begin{aligned}
& {\left[R(t) \sum_{1} R^{*}(t)+\int_{R^{n}} C\left(t, u_{1}\right) V_{1} C^{*}\left(t, u_{1}\right) \Pi_{1}\left(d u_{1}\right)\right]} \\
& \quad=N(t) \\
& {\left[Q(t) \sum_{2} Q^{*}(t)+\int_{R k} D\left(t, u_{2}\right) V_{2} D^{*}\left(t, u_{2}\right) \Pi_{2}\left(d u_{2}\right)\right]} \\
& \quad=M(t)
\end{aligned}
$$

の近似解の構成について考察する.

いま, $(3 \cdot 1)$ 式に $P(t)=Y(t)^{-1}$ なる变数変換をほ ぞこすと,

$$
\begin{aligned}
\dot{Y}(t)= & -A^{*}(t) Y(t)-Y(t) A(t)-Y(t) N(t) Y(t) \\
& +H^{*}(t) M^{-1}(t) H(t) \\
Y\left(t_{0}\right)= & P_{0}^{-1}
\end{aligned}
$$

をえる. ここで， $X(t)$ を $\dot{X}=A(t) X$ の基本解行列 とし, $X\left(t_{0}\right)=I$ (単位行列) とする. 最初に, (3.1), $(3 \cdot 4)$ 式に対する同次方程式を考える $(N(t)=H(t)$ $=0$ ).

これは，システム雑音が存在せず，観測が行なわれ ない場合に対応している. (3·1) 式と (3.4) 式に詨す る一般解は,

$$
P(t)=X(t) C X^{*}(t) ; Y(t)=\left[X^{*}(t)\right]^{-1} C^{-1} X^{-1}(t)
$$

と表わされる，初期条件を考慮すれば， $C=P_{0}$ となる，

次にシステム雑音が存在し, 観測が行なわれない場 合 $(H(t)=0)$ について考光る. このとき，(3.1) 式 は線形の非同次方程式となり, その解は定数変化法に より求めることができて,

$$
\begin{aligned}
& P(t)=X(t)\left[P_{0}\right. \\
& \left.\quad+\int_{t_{0}}^{t} X^{-1}(s) N(s)\left[X^{*}(s)\right]^{-1} d s\right] X^{*}(t)
\end{aligned}
$$

となる。

さらに, システムの雑音が存在しない $(N(t)=0)$ が, 観測が行なわれる場合は，(3-1) 式は非線形の微分方 程式となる。しかし，(3·4)式は線形に帰着され，(3·6) 式を得たと同様にして，

$$
\begin{aligned}
& Y(t)=P^{-1}(t)=\left[X^{*}(t)\right]^{-1}\left[P_{0}^{-1}\right. \\
& \left.\quad+\int_{t_{0}}^{t} X^{-1}(s) H^{*}(s) M^{-1}(s) H(s) X(s) d s\right] X^{-1}(t)
\end{aligned}
$$

を光る。

ここで，観測雑音が大きい場合について考察する. このとき, $M(t)=\varepsilon M_{*}(t)$ とおく.

ただし， $\varepsilon \ll 1$ とし，行列 $M_{*}(t)$ の各要素は有界と する. 万程式 $(3 \cdot 1)$ の解を,

$$
P(t)=P^{0}(t)+\varepsilon P^{1}(t)
$$

なる形で求めよ5。前の議論から $P^{0}(t)$ は $(3 \cdot 6)$ 式

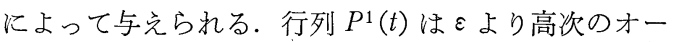
ダの精度で, 万程式,

$$
\begin{aligned}
\dot{P}^{1}(t)= & A(t) P^{1}(t)+P^{1}(t) A(t) \\
& -P^{0}(t) H^{*}(t) M_{*}^{-1}(t) H(t) P^{0}(t) \\
P^{1}\left(t_{0}\right)= & 0
\end{aligned}
$$

によって決定される. この方程式は線形非同次で, そ の解は,

$$
\begin{aligned}
P^{1}(t) & =-X(t)\left[\int_{t_{0}}^{t} X^{-1}(s) P^{0}(s) H^{*}(s) M_{*}^{-1}(s)\right. \\
& \left.\times H^{\prime}(s) P^{0}(s) \cdot\left[X^{*}(s)\right]^{-1} d s\right] X^{*}(t)
\end{aligned}
$$

となる。

かくして, 観測雑音が大きい場合の (3·1) 式の近似 解が構成されたことになる。

同様にして，システム雑音が小さい場合について考 察しょう.

このとき， $N(t)=\varepsilon N_{*}(t)(\varepsilon \ll 1)$ とする．前の議論 々同様にして,

$$
Y(t)=Y^{0}(t)+\varepsilon Y^{1}(t)
$$

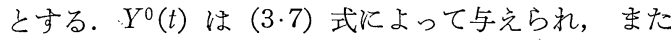

$$
\begin{aligned}
Y^{1}(t)= & -\left[X^{*}(t)\right]^{-1}\left[\int_{t_{0}}^{t} X^{*}(s) Y^{0}(s) N_{*}(s)\right. \\
& \left.\times Y^{0}(s) X(s) d s\right] \cdot X^{-1}(t)
\end{aligned}
$$

である。

したがってシステム雑音が小さい場合の (3·1) 式の 近似解は,

$$
P(t)=Y^{-1}(t)=\left[Y^{0}(t)+\varepsilon Y^{1}(t)\right]^{-1}
$$

によって与えられる.

\section{5. 結論}

一般化確率微分方程式の 1 つの応用として状態推定 問題を扱った．確率連続な状態の推定については，す でに J.R.Stear の論文帛があるが，これは特性関数を 用いて議論するもので, かなり複雑な計算を要する. これに反し，一般化確率微分方程式を用いれば，推定 問題のみならず，(1·1）式で記述されるシステムある いはもっと一般的な非線形のシステムに対して安定性 や可制御性などの議論をすることも可能である。事実, 伊藤形の確率微分方程式に対して考えられた確率的リ 
ヤプーノフ関数をもら少し一般化し, 安定性に関する 十分条件を与えることができる，乙がし，実際のシス テムに対してその条件を検証することは容易ではない。 また，実際上は，非線形システムに関する解析が久か せない、システムをなんらかの方法で線形化すること ができれば，推定過程と最適制御過程を分離すること ができ非常に便利であるが，非線形システムを統一的 に扱うのは困難であるので，ここでは割愛する。

\section{参 考 文 献}

1) R.E. Kalman and R.S. Bucy: New Results in Linear Filtering and Prediction Theory, Trans. ASME, J. Basic Engng., Ser. D. Vol. 83, 95/108 (March-1961)

2) R.E. Kalman, P. L. Falb and M. A. Arbib : Topics in Mathematical System Theory, McGraw-Hill (1969)

3) A. B. Skorokhod: Studies in the Theory of Ran- dom Processes, Addison-Wesley (1965)

4) K. Ito : On Stochastic Differential Equations, 1951 Mem. Amer. Math. Soc., 4

5) I. I. Gichman und A. W. Skorochod: Stochastische Differentialgleichungen, Akademie-Vlg. (1970)

6) И.И. Гихман и А.Я. Аороговцев : Об устойчивости решений стохастических Аифференчиальных уравнений, 1965 УМЖ, T. XVII Nl. 6

7) А.В.Скоғоход : Случайные прочессы с независимыми приращениями, Наука (1964)

8) J.R. Fisher and E. B. Stear: Optimal Nonlinear Filtering for Independent Increment Processes, Part I, II IEEE Trans. Information Theory, IT-34 (1967)

9) Ralph Deutch : Estimation Theory, Prentice-Hall, Inc. (1965)

10) Р. Ш. Липчер и А.Н. Ширяев : Нелинейная Фильтрачия Аиффузионных Марковских Прочессов, ТРУАЫ ордена Ленина Мате. Инс-Та имени Стеклова CIV, 135/180, Наука (1968) 